Pacific Journal of Mathematics

FRATTINI SUBALGEBRAS OF A CLASS OF SOLVABLE LIE 


\section{FRATTINI SUBALGEBRAS OF A CLASS OF SOLVABLE LIE ALGEBRAS}

\section{ERNEST L. STITZINGER}

In this paper the Lie algebra analogues to groups with property $E$ of Bechtell are investigated. Let $\mathfrak{X}$ be the class of solvable Lie algebras with the following property: if $H$ is a subalgebra of $L$, then $\phi(H) \leqq \phi(L)$ where $\phi(L)$ denotes the Frattini subalgebra of $L$; that is, $\phi(L)$ is the intersection of all maximal subalgebras of $L$. Groups with the analogous property are called $E$-groups by Bechtell. The class $\mathfrak{X}$ is shown to contain all solvable Lie algebras whose derived algebra is nilpotent. Necessary conditions are found such that an ideal $N$ of $L \in \mathfrak{X}$ be the Frattini subalgebra of $L$. Only solvable Lie algebras of finite dimension are considered here.

The following notation will be used. We let $N(L)$ be the nil radical of $L$ and $S(L)$ be the socle of $L$; that is, $S(L)$ is the union of all minimal ideals of $L$. If $A$ and $B$ are subalgebras of $L$, let $Z_{B}(A)$ be the centralizer of $A$ in $B$. The center of $A$ will be denoted by $Z(A)$. If $[B, A] \subseteq A$, we let $\operatorname{Ad}_{A}(B)=\{\operatorname{ad} b$ restricted to $A$; for all $b \in B\} . \quad L^{\prime}$ will be the derived algebra of $L$ and $L^{\prime \prime}=\left(L^{\prime}\right)^{\prime}$.

Proposition 1. Let $L$ be a Lie algebra such that $L^{\prime}$ is nilpotent. Then the following are equivalent:

(1) $\phi(L)=0$.

(2) $N(L)=S(L)$ and $N(L)$ is complemented by a subalgebra.

(3) $L '$ is abelian, is a semi-simple L-module and is complemented by a subalgebra.

Under these conditions, Cartan subalgebras of $L$ are exactly those subalgebras complementary to $L$ '.

Proof. Assume (1) holds. Nilpotency of $L^{\prime}$ implies $\phi(L) \supseteqq L^{\prime \prime}$, so $L^{\prime}$ is abelian and may be regarded as an $L / L^{\prime}$-module. We may assume $L^{\prime}=\Sigma \oplus V_{\rho}, V_{\rho}$ indecomposable $L / L^{\prime}$-submodules. If $M$ is a maximal subalgebra of $L$ and if $V_{\rho} \nsubseteq M$, then $M \cap V_{\rho}$ is an ideal of $L$. If $S$ is an $L / L^{\prime}$-submodule of $V_{\rho}$ properly contained between $M \cap V_{\rho}$ and $V_{\rho}$, then $M+S$ is a subalgebra of $L$ properly contained between $M$ and $L$, contradicting the maximality of $M$. Therefore $M$ contains all maximal submodules of $V_{\rho}$ for each $\rho$. Then $\phi(L)=0$ implies the intersection of all maximal submodules of $V_{\rho}$ is zero for each $\rho$. If $V_{1}, \cdots, V_{s}$ are maximal submodules of $V_{\rho}$ with $V_{1} \cap \cdots \cap V_{s}=0$ and are minimal with respect to this property, we have $V=V_{2} \cap \cdots V_{s} \neq 0$ 
and $V \cap V_{1}=0$ so that $V \oplus V_{1}=V_{\rho}$, contradicting indecomposability. Therefore each $V_{\rho}$ is irreducible and $L^{\prime}$ is a completely reducible $L / L^{\prime}$ module and is also a completely reducible $L$-module. Since $L$ is solvable it contains Cartan subalgebras by Theorem 3 of [1]. Let $H$ be a Cartan subalgebra of $L$ and let $L_{0}$ and $L_{1}$ be the Fitting null and one component of $L$ with respect to $H$. Then $L=L_{0}+L_{1}=H+L_{1} \subseteq$ $H+L^{\prime}$ shows $L=H+L^{\prime}$. We claim that $H \cap L^{\prime}=0$. If $H \cap L^{\prime} \neq 0$, then, since $L^{\prime}$ is abelian, $H$ is nilpotent and $L^{\prime}$ is a completely reducible $L$-module, $L^{\prime}$ is a sum of irreducible $H$-modules, $U_{1}, \cdots, U_{o}$,

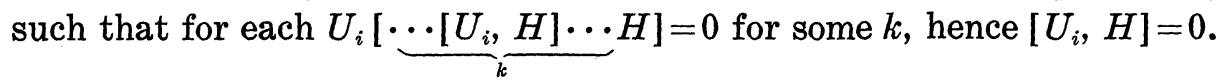

Thus $\left[H, L^{\prime} \cap H\right]=0$. One sees that each $U_{i}$ is a central minimal ideal of $L$, and since $\phi(L)=0, U_{i}$ is complemented by a maximal subalgebra $M$. Therefore $U_{i}$ is a one-dimensional direct summand of $L$, contradicting $U_{i} \subseteq L^{\prime}$. Hence $L^{\prime} \cap H=0$ and $H$ is a complement to $L^{\prime}$ in $L$. Since $[H, H] \subseteq H \cap L^{\prime}=0, H$ is abelian. Any minimal ideal not in $L^{\prime}$ satisfies $[L, A] \subseteq A \cap L^{\prime}=0$, so is central. Therefore $S(L)=$ $L^{\prime}+Z(L)$ and, since $H$ is a Cartan subalgebra, $Z(L) \cong H$. Let $H_{0}$ be a complementary subspace to $Z(L)$ in $H$. One sees that $N(L)=$ $L^{\prime}+Z(L)+\left(N(L) \cap H_{0}\right)=S(L)+\left(N(L) \cap H_{0}\right)$. If $h$ is a nonzero element in $N(L) \cap H_{0}$, ad $h$ is nilpotent but not zero which implies $\left[V_{\rho}, h\right]=V_{\rho}$ for some $V_{\rho} \subseteq L^{\prime}$ and $\left[\cdots[V_{\rho}, \overbrace{h] \cdots h}^{k}]=0\right.$ for some $k$, a contradiction. Thus $S(L)=N(L)$ and $H_{0}$ is a complement. Consequently (1) implies (2).

Assume (2) holds and proceed by induction on the dimension of $L$. Since $L^{\prime} \subseteq N(L)=S(L)$ and minimal ideals are abelian, $L^{\prime}$ is abelian. If every minimal ideal of $L$ is contained in $L^{\prime}$, then $S(L)=L^{\prime}$ and (3) follows. Therefore let $A$ be a minimal ideal of $L$ such that $A \nsubseteq L^{\prime}$. Hence $A \nsubseteq \phi(L)$ and there exists a maximal subalgebra $M$ of $L$ such that $L=M+A$. Since $[L, A] \subseteq A \cap L^{\prime}=0, A$ is central, hence one-dimensional. It follows that $L$ is the Lie algebra direct sum of $M$ and $A$. Since $M$ inherits the condition (2), $M$ satisfies (3) by induction. It now follows that $L$ also satisfies (3).

Assume (3) holds. Then $L^{\prime}$ is a sum of minimal ideals of $L$, which we denote by $A_{1}, \cdots, A_{k}$, and $L=L^{\prime}+H, H$ a subalgebra of $L$. Since $H^{\prime} \subseteq H \cap L^{\prime}=0, H$ is abelian. One sees that $L^{\prime}=\left[L^{\prime}, H\right]$ and, consequently, $A_{i}=\left[A_{i}, H\right]$ for all $i$. Since $Z_{A_{i}}(H)$ is central in $L, Z_{A_{i}}(H)$ is an ideal in $L$ contained in $A_{i}$. Since $Z_{A_{i}}(H) \neq A_{i}, Z_{A_{i}}(H)=0$. It follows that $H$ is its own normalizer, hence is a Cartan subalgebra of $L$. Now $H+A_{1}+\cdots+\hat{A}_{i}+\cdots+A_{k}$ is a maximal subalgebra of $L$ since any containing algebra has a nonzero projection on $A_{i}$ which is ad $H$ stable, hence equal to $A_{i}$. Therefore $\phi(L) \cong H$ and $\phi(L) \subseteq$ $H \cap L^{\prime}=0$. Hence (1) holds. 
That complements to $L^{\prime}$ are Cartan subalgebras is shown in (3) implies (1). That Cartan subalgebras are complements to $L^{\prime}$ is shown in (1) implies (2). This completes the proof of Proposition 1.

THEOREM 1. Let $L$ be a Lie algebra such that $L^{\prime}$ is nilpotent and $\phi(L)=0$. Then, for any subalgebra $M$ of $L, \phi(M)=0$.

Proof. Suppose $L^{\prime} \subseteq M$. Let $H$ be a complement to $L^{\prime}$ in $L$, so $H \cap M$ is a complement to $L^{\prime}$ in $M$. Since $L$ acts completely reducibly on $L^{\prime}$ and $L^{\prime}$ is abelian, $H$ acts completely reducibly on $L^{\prime}$. Then, since $H$ is abelian, $H \cap M$ acts completely reducibly on $L^{\prime}$, hence so does $M$. Therefore $L^{\prime}=M^{\prime} \oplus A$ for some ideal $A$ in $M$ where $M$ acts completely reducibly on $M^{\prime}$ and $A+(H \cap M)$ is a complementary subalgebra of $M^{\prime}$ in $M$. Thus by Proposition $1, \phi(M)=0$.

Suppose $L^{\prime} \nsubseteq M$. Since $M+L^{\prime}$ falls in the preceeding case, we may assume $M+L^{\prime}=L$. Since $L^{\prime}$ is abelian, $L^{\prime} \cap M$ is an ideal in $L, M /\left(L^{\prime} \cap M\right)$ complements $L^{\prime} /\left(L^{\prime} \cap M\right)=\left(L / L^{\prime} \cap M\right)^{\prime}$ in $L /\left(L^{\prime} \cap M\right)$ and $M /\left(L^{\prime} \cap M\right)$ acts completely reducibly in $L^{\prime} /\left(L^{\prime} \cap M\right), M /\left(L^{\prime} \cap M\right)$ is a Cartan subalgebra of $L /\left(L^{\prime} \cap M\right)$. Let $C$ be a Cartan subalgebra of $M$. By Lemma 4 of [1], $C$ is a Cartan subalgebra of $L$. Thus $C$ is a complement to $L^{\prime}$ and $C+\left(L^{\prime} \cap M\right)=M$ since $C \cong M$. Hence $C$ is a complement to $L^{\prime} \cap M$ in $M$. Since $M$ acts completely reducibly on $L^{\prime} \cap M$ and $M^{\prime} \subseteq L^{\prime} \cap M, M$ acts completely reducibly on $M^{\prime}, L^{\prime} \cap M=$ $M^{\prime} \oplus\left(L^{\prime} \cap Z(M)\right)$ and, since $Z(M) \subseteq C, Z(M) \cap L^{\prime} \leqq C \cap L=0$. Therefore $C=M^{\prime}=M$ and $C \cap M^{\prime}=0$. Now $M$ satisfies part (3) of Proposition 1 , hence $\phi(M)=0$.

If $L$ is a solvable Lie algebra it has been shown in [2] that $\phi(L)$ is an ideal of $L$. We look for a condition on the subalgebras of $L / \phi(L)$ which are necessary and sufficient that $L \in \mathfrak{X}$. In order to do this the following concept is introduced.

We shall say that a Lie algebra $L$ is the reduced partial sum of an ideal $A$ and a subalgebra $B$ if $L=A+B$ and for any subalgebra $C$ of $L$ such that $L=A+C$ and $C \sqsubseteq B$ then $C=B$. It is noted that if $A \nsubseteq \phi(L)$, then there exists a $B \neq L$ such that $L$ is the reduced partial sum of $A$ and $B$. On the other hand, if $A \subseteq \phi(L)$ and $L$ is the reduced partial sum of $A$ and $B$, then $B=L$.

Lemma 1. Let $L$ be the reduced partial sum of $A$ and $B$. Then $A \cap B \subseteq \phi(B)$.

Proof. Suppose $C=A \cap B \nsubseteq \phi(B)$. Then $B$ contains a subalgebra $D$ such that $C+D=B$. Then $L=A+B=A+C+D=A+D$. This contradicts the minimality of $B$. 
LEMMA 2. Let $L$ be the reduced partial sum of $A$ and $B$. Then. $\phi(L / A) \simeq A+\phi(B) / A$.

Proof. Since $A \cap B \cong \phi(B), A \cap \phi(B)=A \cap B$. Since $L / A \simeq A+$ $B / A \simeq B / A \cap B, \phi(L / A) \simeq \phi(B / A \cap B) \simeq \phi(B) / A \cap B=\phi(B) / A \cap \phi(B) \simeq$ $A+\phi(B) / A$.

Proposition 2. The following are equivalent for the Lie algebra. $L:$

(1) $L \in \mathfrak{X}$.

(2) For any subalgebra $H$ of $L / \phi(L), \phi(H)=0$.

Proof. Let $L$ satisfy (1) and let $\pi: L \rightarrow L / \phi(L)$ be the natural homomorphism. Then $\phi(\pi(L))=\pi(\phi(L))=0$. Let $\bar{W}$ be a subalgebra. of $L / \phi(L)$ and let $W$ be the subalgebra of $L$ which contains $\phi(L)$ and corresponds to $\bar{W}$. Since $L$ satisfies (1), $\phi(W) \subseteq \phi(L)$. If $\phi(W)=\phi(L)$, then $\phi(\pi(W))=\pi(\phi(W))=\pi(\phi(L))=0$. Suppose then that $\phi(W) \subset \phi(L)$. Then $W$ can be represented as a reduced partial sum $W=\phi(L)+K$. Let $T$ be a subalgebra of $W$ such that $T / \phi(L) \simeq \phi(W / \phi(L))$. If $T / \phi(L) \neq 0$, then $T=T \cap(\phi(L)+K)=(T \cap \phi(L))+(T \cap K)=\phi(L)+(T \cap K)$. Consequently there exists an $x \in T \cap K, x \notin \phi(L)$. Since $\phi(K) \subseteq \phi(L), x \notin \phi(K)$ and there exists a maximum subalgebra $S$ of $K$ such that $x \notin S$. We claim that either $\phi(L)+S=W$ or $\phi(L)+S$ is maximal in $W$. Suppose $\phi(L)+S \neq W$ and let $J$ be a subalgebra of $W$ which contains $\phi(L)+S$. Then $S \subseteq J \cap K$, so, by the maximality of $S$, either $J \cap K=S$ or $J \cap K=K$. If $J \cap K=S$, then $\phi(L)+S=\phi(L)+(J \cap K)=J \cap(\phi(L)+$ $K)=J \cap W=J$. If $J \cap K=K$, then $J \supseteqq K$ and, since $J \supseteqq \phi(L), J \supseteqq$ $\phi(L)+K=W$, hence $J=W$. Consequently there exist no subalgebras of $W$ properly contained between $\phi(L)+S$ and $W$, hence either $\phi(L)+$ $S=W$ or $\phi(L)+S$ is maximal in $W$. If $\phi(L)+S=W$, then $\phi(L)+K$ is not a reduced partial sum which is a contradiction. If $\phi(L)+S$ is maximal in $W$, then $\phi(L)+S / \phi(L) \supseteqq \phi(W / \phi(L)) \simeq T / \phi(L)$. Hence $T \subseteq \phi(L)+S$. Since $S \subseteq \phi(L)+S$ and $x \in T \cap K \subset T \subseteq \phi(L)+S, K=$ $\{S, x\} \subseteq \phi(L)+S$. Then $W=\phi(L)+K \subseteq \phi(L)+S \subseteq W$ implies $\phi(L)+$ $K$ is not a reduced partial sum, a contradiction. Hence $\phi(\bar{W})=$ $T / \phi(L)=0$ and (2) is satisfied.

If $L / \phi(L)$ satisfies (2), then $\pi(\phi(H)) \subseteq \phi(\pi(H))=0$ for every subalgebra $H$ of $L$. Then $\phi(H) \subseteq \phi(L)$ for every subalgebra $H$ of $L$.

Combining Proposition 2 and Theorem 1 we have

THEOREM 2. Let $L$ be a Lie algebra such that $L^{\prime}$ is nilpotent. Then $L \in \mathfrak{X}$.

Theorem 3. Let $L \in \mathfrak{X}$ and let $T$ be a Lie homomorphism of $L$. 
Then $T(\phi(L))=\phi(T(L))$.

Proof. $T(\phi(L))$ is always contained in $\phi(T(L))$ by Proposition 1 in [6]. If $N=$ kernel $T \leqq \phi(L)$, then equality holds by Proposition 2 in [6]. Suppose $N \nsubseteq \phi(L)$. Let $L=N+K$ be a reduced partial sum. Using Lemma 2, $\phi(T(L))=\phi(L / N) \simeq N+\phi(K) / N=T(\phi(K))$. Since $T(N+\phi(L))=T(\phi(L)) \leqq \phi(T(L))=\phi(L / N)=T(N+\phi(K)), N+\phi(L) \subseteq$ $N+\phi(K) \subseteq N+\phi(L)$. Hence $N+\phi(L)=N+\phi(K)$ and $\phi(T(L))=$ $T(\phi(K))=T(\phi(L))$.

THEOREM 4. Let $L \in \mathfrak{X}$. Necessary conditions that an ideal $N$ of $L$ be the Frattini subalgebra of $L$ are that

(1) $\phi\left(\operatorname{Ad}_{N}(L)\right)=\operatorname{Ad}_{N}(\phi(L))$.

(2) There exists a subalgebra $M$ of $L$ such that $M / N \simeq$ $\operatorname{Ad}_{N}(L) / \operatorname{Ad}_{N}(\phi(L))$.

Proof. (1) Let $T$ be the mapping from $L$ into the derivation algebra of $N$ by $T(x)=\operatorname{ad} x$ restricted to $N$ for all $x \in L$. Then $T(\phi(L))=\operatorname{Ad}_{N}(\phi(L))=\phi(T(L))=\phi\left(\operatorname{Ad}_{N}(L)\right)$.

(2) Let $M=Z_{L}(\phi(L))$. Suppose that $M \nsubseteq \phi(L)$ and let $F=L / \phi(L)$ and $A=(M+\phi(L)) / \phi(L)$. Since $\operatorname{Ad}_{\phi(L)}(L) \simeq L / M$ and $\operatorname{Ad}_{\phi(L)}(\phi(L)) \simeq$ $\phi(L) / Z(\phi(L))=\phi(L) / M \cap \phi(L)=(M+\phi(L)) / M, F / A \simeq(L / \phi(L)) /(M+\phi(L) /$ $\phi(L)) \simeq L /(M+\phi(L)) \simeq(L / M) /((M+\phi(L)) / M) \simeq \operatorname{Ad}_{\phi(L)}(L) / \operatorname{Ad}_{\phi(L)}(\phi(L))$. Since $\phi(F)=0$, there exists a subalgebra $D$ in $F$ such that $F$ is the reduced partial sum of $A$ and $D$. Using Proposition 2 and Lemma 1, $A \cap D \leqq \phi(D)=0$, hence $A \cap D=0$. Let $E$ be the subalgebra of $L$ which contains $\phi(L)$ and corresponds to $D$. Then $E / \phi(L) \simeq D \simeq F / A \simeq$ $\operatorname{Ad}_{\phi(L)}(L) / \operatorname{Ad}_{\phi(L)}(\phi(L))$. If $M \cong \phi(L)$, then $\operatorname{Ad}_{\phi(L)}(L) / \operatorname{Ad}_{\phi(L)}(\phi(L)) \simeq$ $(L / M) /(\phi(L) / Z(\phi(L)))=(L / M) /(\phi(L) / M \cap \phi(L))=(L / M) /(\phi(L) / M) \simeq L / \phi(L)$.

Related to part (1) of Theorem 4 are the following results.

THeOREM 5. Let $L \in \mathfrak{X}$ and let $K$ be an ideal of $L$ containing $\phi(L)$. Then $\phi\left(\operatorname{Ad}_{K}(L)\right) \simeq \operatorname{Ad}_{K}(K)$ if and only if $K=\phi(L)+Z(K)$.

Proof. Let $T$ be the Lie homomorphism from $L$ into the derivation algebra of $K$ given by $T(x)=$ ad $x$ restricted to $K$ for each $x \in L$. Then $\quad \phi\left(\operatorname{Ad}_{K}(L)\right)=\phi(T(L))=T(\phi(L))=\operatorname{Ad}_{K}(\phi(L)) \simeq \phi(L) / Z_{\phi(L)}(K)=$ $\phi(L) /(Z(K) \cap \phi(L)) \simeq(\phi(L)+Z(K)) / Z(K)$. If $\phi(L)+Z(K)=K$, then $\operatorname{Ad}_{K}(K) \simeq K / Z(K)=(\phi(L)+Z(K)) / Z(K) \simeq \phi\left(\operatorname{Ad}_{K}(L)\right)$. If $\phi(L)+Z(K) \subset$ $K$, then $\operatorname{Ad}_{K}(K) \simeq K / Z(K) \supset(\phi(L)+Z(K)) / Z(K)=\dot{\phi}\left(\operatorname{Ad}_{K}(L)\right)$.

Theorem 6. Let $L \in \mathfrak{X}$ and let $A$ be an ideal of $L$ contained in $\phi(L)$. Then $\phi\left(\operatorname{Ad}_{A}(L)\right) \simeq \operatorname{Ad}_{A}(A)$ if and only if $\phi(L)=A+Z_{\phi(L)}(A)$. 
Proof. If $\phi(L)=A+Z_{\phi(L)}(A)$, then $\operatorname{Ad}_{A}(A)=\operatorname{Ad}_{A}(\phi(L))=T(\phi(L))=$ $\phi(T(L))=\phi\left(\operatorname{Ad}_{A}(L)\right)$.

Conversely, $\operatorname{Ad}_{A}(L) \simeq L / Z_{L}(A)$ and $Z_{L}(A)+A / Z_{L}(A) \simeq A / Z(A)=$ $\operatorname{Ad}_{A}(A)$. Then $L / Z_{L}(A)+A \simeq \operatorname{Ad}_{A}(L) / \operatorname{Ad}_{A}(A)$ and $\phi\left(L / Z_{L}(A)+A\right) \simeq$ $\phi\left(\operatorname{Ad}_{A}(L) / \operatorname{Ad}_{A}(A)\right)=\phi\left(\operatorname{Ad}_{A}(L)\right) / \operatorname{Ad}_{A}(A)=0$. Hence $\phi(L) \subseteq Z_{L}(A)+A$ and $\phi(L)=Z_{\phi(L)}(A)+A$.

The author wishes to thank the referee for many helpful comments. In particular the present form of Proposition 1 and Theorem 1 are his generalizations to results originally submitted.

\section{REFERENCES}

1. D. W. Barnes, On Cartan subalgebras of Lie algebras, Math Z. 101 (1967) 350-355.

2. D. W. Barnes, and H.M. Gastineau-Hills, On the theory of solvable Lie algebras, Math Z. 106 (1968) 343-354.

3. H. Bechtell, Frattini subgroups and $\phi$-central groups, Pacific J. Math 18 (1966), $15-23$.

4. Elementary groups, Trans. Amer. Math. Soc. 114 (1965), 355-362.

5. N. Jacobson, Lie algebras, Interscience.

6. E. Stitzinger, On the Frattini subalgebra of a Lie algebra, J. Lond. Math. Soc. (to appear)

Received October 8, 1969.

North Carolina State University

Raleigh, North Carolina 


\section{PACIFIC JOURNAL OF MATHEMATICS}

EDITORS

H. SAMELSON

Stanford University

Stanford, California 94305

RichaRd PIERCE

University of Washington

Seattle, Washington 98105
J. DUGUNDJI

Department of Mathematics

University of Southern California

Los Angeles, California 90007

RICHARD ARENS

University of California

Los Angeles, California 90024

\section{ASSOCIATE EDITORS}

E. F. BECKENBACH

B. H. NeumanN

F. WOLF

K. YOSHIDA

\section{SUPPORTING INSTITUTIONS}

UNIVERSITY OF BRITISH COLUMBIA

CALIFORNIA INSTITUTE OF TECHNOLOGY

UNIVERSITY OF CALIFORNIA

MONTANA STATE UNIVERSITY

UNIVERSITY OF NEVADA

NEW MEXICO STATE UNIVERSITY

OREGON STATE UNIVERSITY

UNIVERSITY OF OREGON

OSAKA UNIVERSITY

UNIVERSITY OF SOUTHERN CALIFORNIA
STANFORD UNIVERSITY

UNIVERSITY OF TOKYO

UNIVERSITY OF UTAH

WASHINGTON STATE UNIVERSITY

UNIVERSITY OF WASHINGTON

AMERICAN MATHEMATICAL SOCIETY CHEVRON RESEARCH CORPORATION TRW SYSTEMS

NAVAL WEAPONS CENTER 


\section{Pacific Journal of Mathematics}

May, 1970

Johan Aarnes, Edward George Effros and Ole A. Nielsen, Locally compact

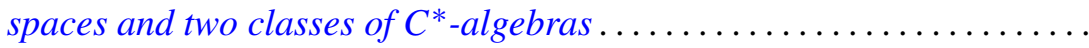

Allan C. Cochran, R. Keown and C. R. Williams, On a class of topological

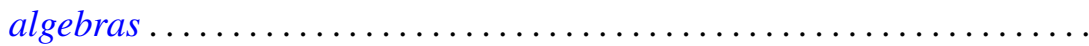

John Dauns, Integral domains that are not embeddable in division rings ....

Robert Jay Daverman, On the number of nonpiercing points in certain

crumpled cubes.....................................

Bryce L. Elkins, Characterization of separable ideals ................

Zbigniew Fiedorowicz, A comparison of two naturally arising uniformities

on a class of pseudo-PM spaces ...........................

Henry Charles Finlayson, Approximation of Wiener integrals of functionals

continuous in the uniform topology ........................

Theodore William Gamelin, Localization of the corona problem ...........

Alfred Gray and Paul Stephen Green, Sphere transitive structures and the

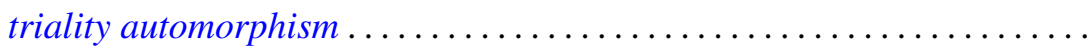

Charles Lemuel Hagopian, On generalized forms of aposyndesis ..........

J. Jakubík, On subgroups of a pseudo lattice ordered group ...............

Cornelius W. Onneweer, On uniform convergence for Walsh-Fourier

series..................................

Stanley Joel Osher, On certain Toeplitz operators in two variables ...

Washek (Vaclav) Frantisek Pfeffer and John Benson Wilbur, On the

measurability of Perron integrable functions............

Frank J. Polansky, On the conformal mapping of variable regions...

Kouei Sekigawa and Shûkichi Tanno, Sufficient conditions for a Riemannian manifold to be locally symmetric ...................

James Wilson Stepp, Locally compact Clifford semigroups ....

Ernest Lester Stitzinger, Frattini subalgebras of a class of solvable Lie

algebras ................................

George Szeto, The group character and split group algebras...

Mark Lawrence Teply, Homological dimension and splitting torsion

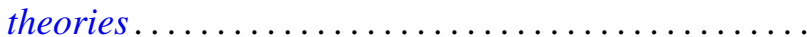

David Bertram Wales, Finite linear groups of degree seven. II ...

Robert Breckenridge Warfield, Jr., An isomorphic refinement theorem for

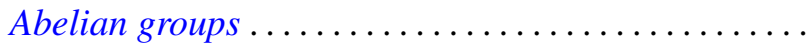

James Edward West, The ambient homeomorphy of an incomplete subspace

of infinite-dimensional Hilbert spaces................

Peter Wilker, Adjoint product and hom functors in general topology ...

Daniel Eliot Wulbert, A note on the characterization of conditional 